\title{
Research on the Execution of Enterprise Management
}

\author{
Zhuoqing Zhang, Ni Xing \\ Nanchang Institute of Science \& Technology, Nanchang, Jiangxi, 330000
}

Keywords: Enterprise Management, Execution Method, Ability Improvement

\begin{abstract}
Management execution is the ability of business management to understand and organize the implementation. In order to grow and develop enterprises, in addition to the correct development strategy, good capital, technology and brand and other factors, the strategy execution is ever more important. Data show that the development of successful enterprises attributed to the planning of enterprises, managers from all levels of execution. Therefore, the execution ability in place reflects the overall quality of enterprises as well as the concept, quality and mentality of management leadership. A good executive department can make up for the lack of decision-making programs, but may lag due to failure of the implementation of the plan, in that executive power is the key to business success.
\end{abstract}

\section{Introduction}

The practice of enterprise development in our country shows that lack of management execution has become a common problem in business management. The concrete manifestation is as follows: On the one hand, during the implementation of the project, the project operation period is too long and the project progress is backward, the tasks assigned by the superior to the subordinate can not be implemented in time, seriously affecting the project progress. On the other hand, the company's management system is not complete, the system implementation status is vague and the responsibility promotion is serious, and the management of the company tends to be "person-controlled" management. The lag of China's enterprise management level has hindered the healthy and steady development of enterprises, the lack of management execution, the failure to implement good solutions, the missed good investment opportunities, and the grave impact on the development of large-scale enterprises. Specifically: First of all, management execution as the basic operation of the company, is the direct embodiment of the company's overall management status. Poor executive ability makes the internal management of the company to slow down, the implementation of the management system is difficult, the lack of effective management experience precipitation, leading to the company in the expansion process of the lack of relatively mature management experience model, increase the project development risk. Second, the lack of execution leads directly to the difficulty of carrying out specific tasks on schedule and effectively. Resulting in the accumulation of follow-up projects, resulting in increased costs, delays in decision-making, capital withdrawal speed and other phenomena, hinder the company's development process, increase the company's operational risk. Finally, the lack of management execution also has a direct impact on the company's ability to respond to customers, partners and companies' external social relations, resulting in increased complaints from owners, deteriorating the environment for cooperation with foreign companies, and the crisis in the relations with government agencies and other social units. As a result, Loss of support for the social environment, the operation greatly increased the difficulty of bringing the company operating crisis [1].

\section{Reasons for the lack of implementation of the enterprise management in China}

The insufficiency of execution of enterprise management in our country stems from the imperfect system of enterprise system and the corporate culture and organizational structure behind the system. In the system of management execution system, as a comprehensive reflection of the speed and effectiveness of the organization and management system in response to the task 
execution and change, the reasons for the lack of management execution are as follows [2]:

Lack of systematic and trustworthy system of management system to ensure the normative operation of the company, the shareholders lack of trust in the operation of the company, can not be effectively authorized; system is incomplete, there is no formation of an effective system of systems, at the same time, the mutual match between the system also exists Problems, can not form an organic link; part of the system is not high applicability, the directive set too high requirements, beyond the efforts of staff can reach the level of non-compliance with the goals set should be followed, "accessibility" and "rationality" in principle.

The lack of appropriate evaluation mechanism, making the company's middle-level cadres lack of enthusiasm for the implementation of standardized management and motivation; the lack of necessary incentives to enable employees to implement the standard management is deducted money and binding work behavior Wrong thinking The implementation of the system lacks a strong monitoring mechanism for implementation, there is no specific leadership and responsible departments to bear the responsibility of supervision and implementation of the system, the effectiveness of the assessment of the implementation of the system is not enough, the implementation of the relationship with the interests of employees is low, the lack of adequate system implementation of the masses basis. It can not be modified and issued in time according to the development of the company. The outdated system also affects the authority of the system. Some systems are not perfect enough to meet the requirements of the company's business development. In addition, in addition to the above problems in the system of enterprise management, the lack of management execution and its corporate culture and organizational structure and other aspects of the deep-seated reasons are: the company's top management team and the company's investor is not clear division of responsibilities. Most of China's corporate chairman is the company's investors, the highest decision-makers, managers and other roles in one, making the company's top management team becomes the executive director of executive direction, and therefore can not effectively fulfill the senior management team should Some management functions; unclear division of responsibilities between company funders and top management, unclear decision-making power, and corporate governance structure problems; the chairman of the board from the centralized management style of entrepreneurship to the standardized level-by-level Type of management style change, the conflict between centralization and authorization will also be reflected in the transformation process. The lack of institutional management and standardized management of the cultural foundation. Emphasize that\% of the internal work of enterprises according to law, and lead by example, is the responsibility of managers at all levels, but there are understanding within the enterprise deviation from top to bottom. Start-ups consider how to use policies, but with the expansion of the scale of enterprises, enterprises should obtain long-term development, and only go from the inside out to standardization and take the initiative to comply with internal and external rules [3].

Internal management team communication, coordination is not enough, the overall lack of combat capability. The management style of the general manager does not match with the awareness of the middle managers, and the implementation of the middle managers is poor, which makes the general manager tired of dealing with the daily affairs of the company and reduces the management efficiency. The uneven structure of the senior management team skills is evident in many companies' day-to-day business and project implementation. The organizational structure of the company is not clear and unstable, and the flow of instruction information is chaotic. The lack of a clear and stable organizational structure led to the frequent changes in the chain of command, seriously affecting the seriousness of corporate governance.

\section{Improve the implementation of enterprise management strategies}

Top management should have strong execution. Execution is a "number one" project. The deep understanding of execution and the determination to enforce it are the responsibilities of top management. Managers must pay attention to implementation. The modern management theory points out that the thoughts, ideas, values and behaviors of top managers play a direct and positive 
role in guiding the ideological behavior of all employees in the enterprise. Demonstration and motivation, and the primary condition for improving executive ability are the need to establish a strong executive force Senior management team, the use of efficient and pragmatic and one line of business philosophy to guide the production and operation of enterprises, human resources, performance and other specific management measures for the formulation and implementation.

Complete implementation of the framework. A sound management structure in the power structure should have a reasonable and appropriate mode of design and democratic management, which can give full play to the initiative, enthusiasm and creativity of employees. However, the binding power of management is relatively weak and the efficiency is relatively low. The centralized management mode Higher efficiency, managers can communicate flat, but it is easy to make the rights of managers unknown, unclear responsibilities. Business managers should start from the actual business, according to the characteristics of the industry, the quality of staff and industries engaged in the adoption of appropriate management, but no matter what kind of management model, business leaders must strive to create a strong implementation of the atmosphere, the formation of the implementation framework, the modern enterprise One of the operational characteristics is the need for inter-departmental communication and coordination, building an implementation framework is conducive to improving coordination among departments. Through the establishment of a smooth internal communication channel, a standardized and rule-based management mechanism of "system management rather than human control" will be formed [4].

Establish a smooth implementation of the process. Enterprise implementation process includes strategic processes, personnel processes and operational processes, of which the staff process is the key to the implementation process of enterprises, is the top priority. Personnel process is not only to play a talent team in the role of enterprises, more importantly, for the implementation of corporate strategy to find talent for the development of enterprises reserve talent. The unobstructed process of establishing a staff is that we must use a combination of material and spiritual means to provide them with a guarantee mechanism that can play an incentive and restraint role and maximize the initiative and creativity of employees so as to exert the role of a process. Another important role of the staff process is to identify those who have poor performance in a timely manner to identify who should change jobs and develop skills training programs for them.

Create a good executive culture. Some scholars think that top management is the preacher and interpreter of corporate culture. Many enterprise managers also think that executive culture can bring strong execution to the enterprise, neglect the re-promotion of team manager's execution ability, the perfection of executive process and the redevelopment and execution culture of executive tools are the comprehensive products after the executive ability of enterprises is improved. The formation of executive culture is not complicated, and does not need to be deliberately pursued. When enterprises persistently improve the managers' abilities, build an implementation framework, improve the implementation process, the development of implementation tools, companies have begun to establish its own unique corporate culture of execution laid the foundation.

Improve the business training system. Knowledge and skills are the basis for improving executive ability. Enterprises must maintain the basis of execution through the establishment of a systematic and scientific learning and training system. First of all, enterprises should make reasonable human resource planning, allocate suitable personnel in suitable positions, adjust or dismiss incompetent personnel and provide training opportunities and provide suitable resources support according to job responsibilities. Second, employees must strengthen self-learning and improve their own quality. Third, enterprises should actively carry out "group learning" and promote "full-time learning" by carrying out such activities as learning organization. They emphasize the development of the intelligence of the members of the organization through planning, Stage of learning, rotation training, work pressure and other means to help employees improve their ability to work [5].

Enterprise development needs funds, technology, personnel, but also need to develop business support point, the support is not a person, but a person-centered team, the team is coordinated, how 
to play its role, first of all depends on the team The core figure, secondly, depends on the team's value orientation and overall quality. Good and bad teamwork will have a crucial impact on the team's work efficiency and quality of work, as well as the speed, size and quality of the organization's growth. How to train the team, give play to the team's overall advantages, and create an effective management execution force, we must make efforts from both the subjective and objective aspects. Objectively speaking, efforts should be made to create a general atmosphere of "teamwork" and stress the principle of "three acts" in work, that is, act according to procedures, act according to systems and act according to objective laws. The person who carries out the procedure is responsible for the "affairs" rather than being responsible for "the people," diluting the individual's role and emphasizing the terminal that accomplishes the work directly by abiding by the same rules. Subjectively speaking, all team members should enhance their overall concept and overall awareness. Instead of emphasizing "self-centeredness," they emphasize "the overall interest is the guide." In the event of uncoordination, we should "seek common ground while reserving differences." Find common ground. At work, we should carry forward the spirit of "someone is responsible for my obedience and nobody is responsible for what I am responsible for." However, we should not advocate blindness and offside responsibility. In particular, those who do not do their jobs well and who are blindly and offsidely responsible will seriously affect the execution of management.

\section{Conclusions}

Good execution of enterprise management can make enterprises adapt to the needs of reform and development, enhance their ability to resist risks, and ensure the healthy, steady and sustainable development of the enterprise, which is crucial to the future development of the enterprise. This article gives a brief overview of the existing problems in the execution of the enterprise management, and puts forward some concrete solutions in the hope of improving the execution ability of some enterprises.

\section{References}

[1] Kong Fanjuan, Li Rui. Discussion on the Influencing Factors of Strategic Executive Power in Business Management [J]. Human Resource Development. 2015 (22):54 58.

[2] Luo Jianqiang. Diligent "internal strength" to enhance the implementation of enterprise strategy [J]. Human Resource Management. 2013 (07) :77 81.

[3] Li Xiangqian. On the Influencing Factors of Enterprise Strategy Execution Power [J]. China Ocean Engineering and Technology, 2008 (06) :12 14.

[4] Lin Yueqin. How to improve the strategic execution of enterprises [J]. Science decision-making. 2006 (03) :162 178.

[5] Zhang Xinli. On the importance of the implementation of the strategy in business management [J] .2010 (33) :82 88. 